\title{
openheart Right ventricular mechanics and contractility after aortic valve replacement surgery: a randomised study comparing minimally invasive versus conventional approach
}

\author{
Nashmil Hashemi, ${ }^{1,2}$ Jonas Johnson, ${ }^{3}$ Lars-Åke Brodin, ${ }^{3}$ \\ Andreia Gomes-Bernardes, ${ }^{2}$ Ulrik Sartipy, ${ }^{4,5}$ Peter Svenarud, ${ }^{4,5}$ Magnus Dalén, ${ }^{4,5}$ \\ Magnus Bäck, ${ }^{6}$ Mahbubul Alam, ${ }^{1}$ Reidar Winter ${ }^{1}$
}

\begin{abstract}
- Additional material is published online only. To view please visit the journal online (http://dx.doi.org/10.1136/ openhrt-2018-000842).
\end{abstract}

To cite: Hashemi N, Johnson J, Brodin L-Å, et al. Right ventricular mechanics and contractility after aortic valve replacement surgery: a randomised study comparing minimally invasive versus conventional approach. Open Heart 2018;5:e000842. doi:10.1136/ openhrt-2018-000842

Received 20 April 2018 Revised 3 May 2018 Accepted 29 May 2018

\section{Check for updates}

For numbered affiliations see end of article.

Correspondence to Dr Nashmil Hashemi; nashmil. hashemi@ki.se

\section{ABSTRACT}

Objective Minimally invasive aortic valve

replacementsurgery (MIAVR) is an alternative surgical technique to conventional aortic valve replacement surgery (AVR) in selected patients. The randomised study Cardiac Function after Minimally Invasive Aortic Valve Implantation (CMILE) showed that right ventricular (RV) longitudinal function was reduced after both MIAVR and AVR, but the reduction was more pronounced following AVR. However, postoperative global RV function was equally impaired in both groups. The purpose of this study was to explore alterations in RV mechanics and contractility following MIAVR as compared with AVR.

Methods A predefined post hoc analysis of CMILE consisting of 40 patients with severe aortic valve stenosis who were eligible for isolated surgical aortic valve replacement were randomised to MIAVR or AVR. RV function was assessed by echocardiography prior to surgery and 40 days post-surgery.

Results Comparing preoperative to postoperative values, RV longitudinal strain rate was preserved following MIAVR $(-1.5 \pm 0.5$ vs $-1.5 \pm 0.41 / \mathrm{s}, \mathrm{p}=0.84)$ but declined following AVR $(-1.7 \pm 0.3$ vs $-1.4 \pm 0.3$ $1 / \mathrm{s}, \mathrm{p}<0.01)$. RV longitudinal strain reduced following AVR $(-27.4 \pm 2.9 \%$ vs $-18.8 \% \pm 4.7 \%, p<0.001)$ and MIAVR ( $-26.5 \pm 5.3 \%$ vs $-20.7 \% \pm 4.5 \%, p<0.01)$. Peak systolic velocity of the lateral tricuspid annulus reduced by $36.6 \%$ in the AVR group $(9.3 \pm 2.1$ vs $5.9 \pm 1.5 \mathrm{~cm} / \mathrm{s}$, $\mathrm{p}<0.01)$ and $18.8 \%$ in the MIAVR group $(10.1 \pm 2.9$ vs $8.2 \pm 1.4 \mathrm{~cm} / \mathrm{s}, \mathrm{p}<0.01$ ) when comparing preoperative values with postoperative values.

Conclusions RV contractility was preserved following MIAVR but was deteriorated following AVR. RV longitudinal function reduced substantially following AVR. A decline in RV longitudinal function was also observed following MIAVR, however, to a much lesser extent.

\section{INTRODUCTION}

Minimally invasive aortic valve replacement (MIAVR) surgery has in selected patients become an alternative approach to

\section{Key questions}

What is already known about this subject?

- It is well known that right ventricular (RV) longitudinal function as assessed by tricuspid annulus peak systolic excursion (TAPSE) and peak systolic velocity of lateral tricuspid annulus (RVS) decline following aortic valve replacement (AVR) surgery.

- In some studies, this phenomenon has been regarded as reduced global RV function, while other studies have reported preserved global RV function despite a reduction in TAPSE and RVS.

- The number of studies analysing the impact of minimally invasive aortic valve replacement (MIAVR) surgery on TAPSE and RVS are limited: one study did not show any decline in TAPSE and RVS following MIAVR, ${ }^{1}$ while the Cardiac Function after Minimally Invasive Aortic Valve Implantation (CMILE) study showed a decline in TAPSE and RVS following MIAVR.

What does this study add?

- Most of previous studies have been analysing the impact of AVR or MIAVR on RV function by methods that are load dependent. Few studies have assessed the impact of AVR/MIAVR on RV function by methods that are load independent.

- In our randomised study, we aim to analyse and compare the impact of AVR and MIAVR on RV performance with various load-dependent and load-independent modalities.

- The main finding of our study is that both AVR and MIAVR modified RV mechanics. However, intrinsic RV contractility quantified by strain rate was preserved following MIAVR, while it was deteriorated following AVR.

conventional full sternotomy aortic valve replacement (AVR) surgery. A number of studies have compared the efficacy, long-term results, clinical outcomes and patient satisfaction between AVR and MIAVR in the treatment of severe aortic valve stenosis. ${ }^{2-4}$ However, less has been explored regarding the impact of 


\section{Key questions}

How might this impact on clinical practice?

- Assessment of RV performance by echocardiography should include both load-dependent and load-independent metrics. Furthermore, evaluation of RV function should be incorporated in decision making and risk stratification of patients undergoing aortic valve surgery.

these surgical techniques on right ventricular (RV) function. Evaluation of RV performance is of eminent clinical importance in the management of patients with a variety of cardiac diseases. It has been shown that RV function is one of the major determinants of symptoms and survival in patients with heart failure ${ }^{56}$ Furthermore, it has been reported that perioperative $\mathrm{RV}$ dysfunction is a predictor of morbidity and mortality following AVR. ${ }^{7-10}$

Previous studies have consistently reported a reduction in RV longitudinal function assessed by tricuspid annulus peak systolic excursion (TAPSE) and peak systolic velocity of lateral tricuspid annulus (RVS) following AVR ${ }^{11-13}$ However, conclusions drawn from these findings regarding global RV function have been divergent. While some have proposed a reduction in global RV function as assessed by TAPSE and RVS, ${ }^{11}$ others have reported a preserved global RV function despite a reduction in TAPSE and RVS. ${ }^{13}$ These conflicting conclusions are reflecting several aspects of: (i) the complex geometry of the RV, making evaluation of global RV function complicated; (ii) the complex pathophysiology leading to decreased RV longitudinal function following AVR; and (iii) the commonly used measurements in previous studies (eg, TAPSE, RVS and fractional area change (RV-FAC) ) are not pure measures of myocardial contractility but rather strongly dependent on the loading conditions and mechanical influences from the loss of pericardial support and adherences. While TAPSE and RVS are measures of RV mechanics, it has been shown that strain rate (RV longitudinal strain rate (RV-LSR)) is the best measure of myocardial contractility. ${ }^{14} 15$ There are only a few studies addressing impact of MIAVR on $\mathrm{RV}$ function. Unsworth et $a l^{1}$ have reported preserved longitudinal function as assessed by TAPSE following MIAVR as compared with AVR. The randomised study Cardiac Function after Minimally Invasive Aortic Valve Implantation (CMILE) showed that TAPSE was reduced following AVR as well as after MIAVR, but the reduction was more pronounced following AVR. However, global RV function as assessed by RV-FAC was equally impaired in both groups postoperatively. ${ }^{16}$ In the present study, which is a predefined post hoc analysis of CMILE, we aimed to further evaluate alterations in RV function in terms of both RV mechanics and contractility by assessing the velocity of the longitudinal displacement of the RV free wall, RV longitudinal strain (RV-LS) and RV-LSR. We hypothesise that the impact on RV function from MIAVR compares favourably to AVR due to less trauma applied during surgery.
MATERIALS AND METHODS

\section{Study design}

The CMILE study was designed as a randomised, singlecentre, open-label clinical trial. The methods and design of CMILE study has been previously described elsewhere. ${ }^{16}$ In brief, patients assigned to aortic valve replacement surgery at Karolinska University Hospital in Stockholm, Sweden, were eligible for inclusion into the study. Forty patients were randomised either to AVR or MIAVR in a 1:1 ratio, between October 2013 and July 2015.

All patients provided a written informed consent. The study is "Post-results" of CMILE study, registered at clinical trials.gov (NCT01972555).

\section{Patient population}

The inclusion criteria were (1) age $\geq 18$ years; (2) severe AS: defined as peak velocity $\geq 4 \mathrm{~m} / \mathrm{s}$, mean gradient $\geq 40 \mathrm{~mm}$ $\mathrm{Hg}$, aortic valve area (AVA) $<1.0 \mathrm{~cm}^{2}$ or indexed AVA $<0.6 \mathrm{~cm}^{2}$ by Doppler echocardiography in combination with two-dimensional echocardiographic morphology of severe valvular stenosis; (3) referred for surgical replacement of severe AS in adherence to current guidelines ${ }^{17}$; and (4) sinus rhythm.

Patients were excluded if they had (1) reduced left ventricular ejection fraction (LVEF) $<45 \%$ ); (2) previous cardiac surgery; (3) concomitant other severe valvular heart disease; (4) coronary artery disease (CAD) requiring surgical intervention; and/or (5) urgent or emergent surgery.

Preoperatively, all patients were investigated by coronary angiography for evaluation of coexisting CAD. Echocardiography was performed within 1 week before surgery and 40 days post-surgery.

Patients were analysed as treated (the patients who crossed over from one group to the other group were analysed in the new group).

\section{SURGICAL TECHNIQUES}

\section{Minimally invasive aortic valve replacement surgery}

MIAVR was performed via upper partial ministernotomy approach. Approximately a $6 \mathrm{~cm}$ vertical skin incision over the upper part of the sternum was applied. A partial $\mathrm{J}$-shaped incision was extended into the right third intercostal space. A small vertical pericardial incision was applied anterior to the ascending aorta and subsequently aortic valve was revealed. CE-marked and FDA-approved mechanical and bioprosthetic aortic valves (conventional stented or sutureless bioprosthesis) were implanted. The pericardial incision was closed at the end of the procedure.

Cardiopulmonary bypass was established through a central arterial and peripheral venous cannulation. Antegrade crystalloid cardioplegia solution was used.

\section{Conventional aortic valve replacement surgery}

AVR was performed via full median sternotomy. A complete pericardial incision was performed. Myocardial 
protection was performed using antegrade and/or retrograde cold blood cardioplegia. A bioprosthetic or mechanical aortic valve prosthesis was implanted. Pericardium was not closed at the end of operation.

\section{Echocardiographic assessment}

Comprehensive transthoracic echocardiography examination was performed according to the recent guidelines. ${ }^{18}$ All examinations were performed on a Vivid E9 (GE, Healthcare, Horten, Norway). The images were stored digitally for subsequent analysis. An experienced echocardiographer, who was blinded to all clinical data, assessed all analysis off-line using commercially available software EchoPAC V.201 (GE Vingmed Ultrasound AS, Horten, Norway).

\section{Right heart}

RV size and function was quantified in accordance with American Society of Echocardiography Guidelines. ${ }^{18}$

RV-LS and RV-LSR were obtained by Speckle Tracking Echocardiography (STE) from RV-focused four-chamber view. The gain setting and the depth of cine loops were optimised in order to achieve a frame rate of at least $50 / \mathrm{s}$. Subsequent analysis was conducted with dedicated, available commercial software for 2D strain analysis on EchoPAC V.201. The region of interest (ROI) was placed over the RV free wall. The width of the ROI was optimised in order to limit it to the myocardium. End systole was predefined by the software used for strain analysis, in which an automatic estimate of aortic valve closure was used. The software automatically calculated segmental and the global RV-LS and RV-LSR. RV stroke volume was calculated according to the existing guidelines. ${ }^{19}$

RVS was measured as the maximal systolic velocity of the RV free wall displacement at the level of tricuspid annulus using cardiac state diagram. ${ }^{20}$

Right atrial (RA) volume was assessed by tracing the RA endocardial border in a dedicated apical four-chamber view and by using single plane disk summation. Systolic pulmonary artery pressure was calculated by measuring the maximal tricuspid regurgitant velocity and estimation of RA pressure.

\section{Left heart}

Left ventricular (LV) size and function was assessed in accordance to current guidelines. ${ }^{18}$ LVEF was assessed using modified Simpson's biplane. LV longitudinal strain was obtained by STE. LV diastolic function was assessed by measuring peak early diastolic transmitral flow velocity (LVE) and peak late diastolic transmitral flow velocity (LVA). Deceleration time of LVE derived from Doppler recordings of the mitral inflow. The ratio of LVE/LVA was calculated. Left atrial (LA) volume was assessed with the same method as RA volume.

\section{Automated analysis of the right ventricle}

The myocardial traces from the tissue Doppler imaging (TDI) recordings were imported to a software called GHLab (Gripping Heart AB, Stockholm, Sweden), where the phases of the cardiac cycle and velocities were automatically identified by the software. The cardiac mechanical time events are defined according to the Dynamic Adaptive Piston Pump principle that describes the heart as a mechanical pump controlled by its inflow. ${ }^{212}$ The movement of the atrioventricular plane initiates the mechanical functioning of the heart and, therefore, the atrial contraction is considered as the starting point of the cardiac cycle. The different phases in the cardiac cycle are defined by shifts in myocardial mechanical work rather than by the opening or closure of the valves. The terms pre-ejection and post-ejection are used instead of isovolumic contraction and relaxation. ROI is chosen at the level of tricuspid annulus of RV free wall. The six phases of the cardiac cycle were identified as: atrial contraction, pre-ejection period, ventricular ejection time, post-ejection period, rapid filling/early diastole and slow filling/ diastasis.

The duration of each phase, peak myocardial velocities during right ventricular ejection (RVS), tricuspid annular early diastolic velocity $\left(\mathrm{RVE}^{\prime}\right)$ and tricuspid annular late
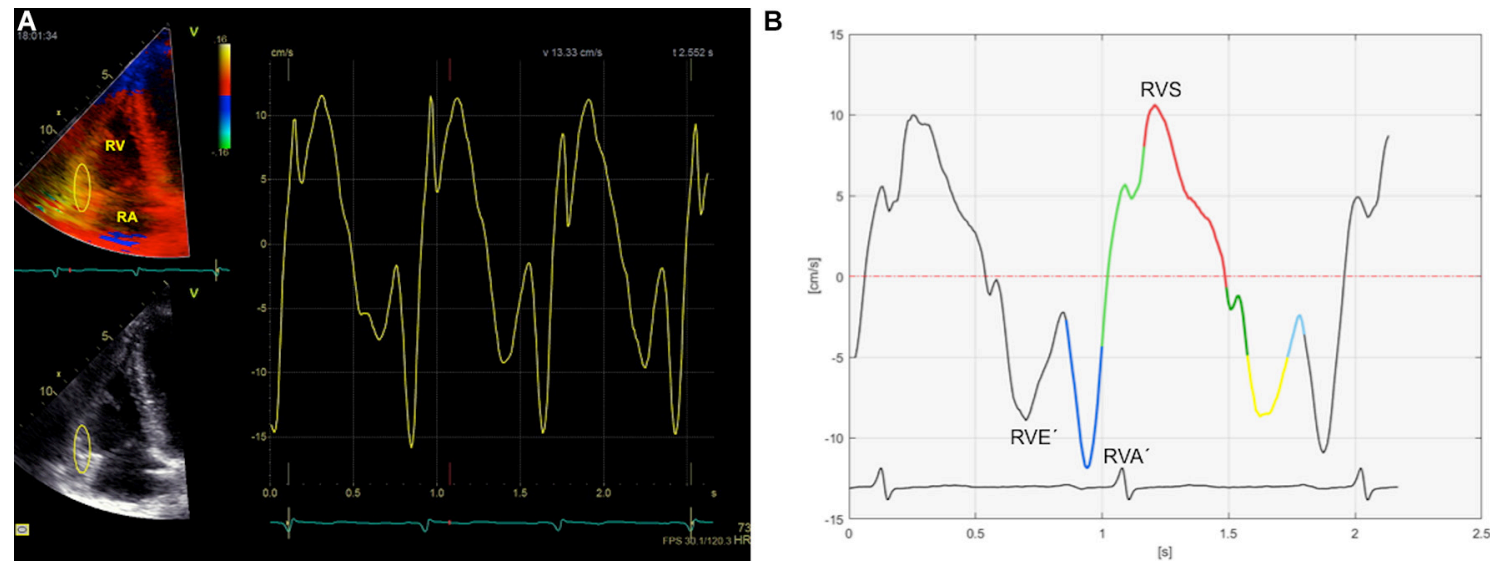

Figure 1 Automated analysis of cardiac velocities and time intervals: positioning region of interest (A); the curves in GHLab (B). 
diastolic velocity (RVA') were obtained from the software (GHLab) as shown in figure 1. Subsequently right ventricular index of myocardial performance (RIMP) and ratio between $\mathrm{E}^{\prime} / \mathrm{A}^{\prime}$ were calculated.

\section{Statistical methods}

The primary endpoints of this study have previously been reported in the CMILE study. ${ }^{16}$ Statistical analyses were performed using SPSS (V.22) and MATLAB. Continuous data are reported as means \pm SD. Categorical data are presented as frequencies or percentages. Predata and postdata within each group were compared by paired Student's t-test or Wilcoxon signed-rank test whenever suitable. Predata and postdata between AVR and MIAVR were compared using independent samples t-test or non-parametric independent sample whenever suitable. Categorical data were compared using $\chi^{2}$. All tests were performed as two-sided tests with a significance level of 0.05 .

\section{RESULTS}

A total of 40 patients were enrolled in the study, of which 20 patients were randomised to AVR and 20 patients to MIAVR. One patient who was randomised to MIAVR was converted to AVR intraoperatively due to difficulties in cardioplegia. Two patients who were randomised to AVR died after surgery (1) at 2 days post-surgery due to acute pancreatitis and multiorgan disease; and (2) at 4 days postsurgery due to undiagnosed liver cirrhosis and acute hepatic failure. These patients were excluded from the analysis. Finally, 19 patients were analysed in each group.
Baseline characteristics are presented in (table 1). Mean age was slightly but not significantly lower in the patients undergoing MIAVR compared with patients undergoing AVR. The number of patients with diabetes and previous stroke was slightly higher in the AVR arm but without reaching the statistical significance level. However, the proportion of patients suffering from renal impairment, and hyperlipidaemia was higher in the MIAVR group but did not reach statistical significance. There was no significant difference in patients' medication between AVR and MIAVR groups, neither before surgery nor after surgery (online supplementary appendix 1).

In the AVR arm, 14 patients received bioprostheses: Edwards Perimount $(\mathrm{n}=10)$, St Jude Trifecta $(\mathrm{n}=2)$ and Medtronic Hancock II ( $\mathrm{n}=2)$, and five patients received a mechanical prosthesis: St Jude Regent $(n=4)$ and Sorin Slimline $(\mathrm{n}=1)$. In the patients undergoing MIAVR, 14 bioprostheses were also implanted, where $50 \%$ were sutureless: Sorin Perseval S $(\mathrm{n}=6)$ and Edwards Intuity $(\mathrm{n}=1)$, and five mechanical prostheses were implanted: Sorin Bicarbon Slimline $(n=5)$. The mean valve size was $23 \mathrm{~mm}$ in both AVR and MIAVR groups. The cardiopulmonary bypass time was longer in the MIAVR group compared with AVR group (113.4 \pm 36 vs $87.4 \pm 28.2$ min, $\mathrm{p}=0.04)$. Aortic cross clamp time was similar in the MIAVR and AVR groups $(80 \pm 21.8$ vs $82.9 \pm 26.9 \mathrm{~min}$, $\mathrm{p}=0.72$ ). The 30 -day mortality was $10 \%$ in the AVR group and none in the MIAVR group. The perioperative/postoperative data are presented in supplementary appendix 2.

Table 1 Comparison of baseline clinical characteristics between patients undergoing AVR and patients undergoing MIAVR

\begin{tabular}{lllll}
\hline Characteristics & AVR $(\mathbf{n = 1 9 )}$ & MIAVR $(\mathbf{n = 1 9 )}$ & P values & Total $(\mathbf{n = 3 8 )}$ \\
\hline Male & $11(57.9 \%)$ & $12(63.2 \%)$ & 0.74 & $23(60.5 \%)$ \\
\hline Age (years) & $70.8 \pm 8$ & $67.3 \pm 9$ & 0.22 & $70 \pm 9$ \\
\hline Hight $(\mathrm{cm})$ & $170.9 \pm 9$ & $172.8 \pm 9$ & 0.55 & $172 \pm 9$ \\
\hline Wight $(\mathrm{kg})$ & $75.5 \pm 12$ & $84.1 \pm 19$ & 0.15 & $81 \pm 16$ \\
\hline Hypertension & $13(68.4 \%)$ & $12(63.2 \%)$ & 0.73 & $25(65.8 \%)$ \\
\hline Diabetes & $5(26.3 \%)$ & $4(21.1 \%)$ & 0.70 & $9(23.7 \%)$ \\
\hline Previous stroke & $2(10.5 \%)$ & 0 & 0.15 & $2(5.3 \%)$ \\
\hline Hyperlipidaemia & $6(31.6 \%)$ & $8(42.1 \%)$ & 0.50 & $14(36.8 \%)$ \\
\hline Previous PCl & 0 & $1(5.3 \%)$ & 0.31 & $1(2.9 \%)$ \\
\hline Renal failure & 0 & $2(10.5 \%)$ & 0.15 & $2(5.3 \%)$ \\
\hline Systolic blood pressure $(\mathrm{mm} \mathrm{Hg})$ & $132 \pm 13$ & $134 \pm 20$ & 0.70 & $133 \pm 16$ \\
\hline Diastolic blood pressure $(\mathrm{mm} \mathrm{Hg})$ & $72 \pm 10$ & $73 \pm 8$ & 0.89 & $73 \pm 9$ \\
\hline ECG & & & & \\
\hline LBBB & $2(10.5 \%)$ & 0 & 0.16 & $2(5.3 \%)$ \\
\hline RBBB & $1(4.8)$ & $1(4.8)$ & 0.97 & $2(5.3 \%)$ \\
\hline QRS duration $(\mathrm{ms})$ & $95.7 \pm 16.8$ & $93.4 \pm 10.3$ & 0.64 & $94.6 \pm 13.8$ \\
\hline Heart rate (beats/min) & $66 \pm 13$ & $69 \pm 9$ & 0.60 & $67 \pm 11$ \\
\hline
\end{tabular}

AVR, aortic valve replacement surgery; LBBB, left bundle branch block; MIAVR, minimally invasive aortic valve replacement surgery; PCI, percutaneous coronary intervention; QRS, the second wave in ECG; RBBB, right bundle branch block. 
Table 2 Preoperative baseline and postoperative echocardiographic RV measurements in the two surgical groups

Pre aortic valve surgery

Post aortic valve surgery

\begin{tabular}{|c|c|c|c|c|c|c|}
\hline \multirow[b]{2}{*}{ Variables } & \multicolumn{2}{|c|}{ Pre aortic valve surgery } & \multicolumn{2}{|c|}{ Post aortic valve surgery } & \multicolumn{2}{|l|}{$P$ values } \\
\hline & AVR & MIAVR & AVR & MIAVR & $\begin{array}{l}\text { Between } \\
\text { the groups } \\
\text { (preoperative)† }\end{array}$ & $\begin{array}{l}\text { Between } \\
\text { the groups } \\
\text { (postoperative) } \dagger\end{array}$ \\
\hline $\begin{array}{l}\text { RV longitudinal strain } \\
(\%)\end{array}$ & $-27.4 \pm 2.9$ & $-26.5 \pm 5.3$ & $-18.8 \pm 4.7^{\star \star \star}$ & $-20.7 \pm 4.5^{\star \star}$ & 0.56 & 0.25 \\
\hline RV strain rate (1/s) & $-1.7 \pm 0.3$ & $-1.5 \pm 0.5$ & $-1.4 \pm 0.3^{\star \star}$ & $-1.5 \pm 0.4$ & 0.32 & 0.53 \\
\hline $\operatorname{RVE}^{\prime}(\mathrm{cm} / \mathrm{s})$ & $8.4 \pm 2.9$ & $7.1 \pm 2.1$ & $4.7 \pm 2.9^{\star \star}$ & $4.7 \pm 2^{\star \star \star}$ & 0.14 & 0.99 \\
\hline $\mathrm{RVA}^{\prime}(\mathrm{cm} / \mathrm{s})$ & $12.2 \pm 3.5$ & $12.2 \pm 2.9$ & $4.9 \pm 1.9^{\star \star}$ & $9 \pm 3.2^{\star \star \star}$ & 0.97 & $<0.001$ \\
\hline $\mathrm{RV} \mathrm{E}^{\prime} / \mathrm{A}^{\prime}$ & $0.84 \pm 0.6$ & $0.59 \pm 0.2$ & $1.1 \pm 0.9$ & $0.55 \pm 0.3$ & 0.16 & 0.04 \\
\hline RV stroke volume $(\mathrm{mL})$ & $62.5 \pm 18.2$ & $63.4 \pm 13.1$ & $56.6 \pm 15.2$ & $67.7 \pm 10.3$ & 0.64 & 0.014 \\
\hline POP (ms) & $100.8 \pm 27$ & $103.7 \pm 25$ & $116.2 \pm 30$ & $100.3 \pm 35$ & 0.74 & 0.19 \\
\hline RVOT prox (mm) & $30.1 \pm 3.2$ & $30.2 \pm 3.9$ & $29.2 \pm 3.9$ & $29.2 \pm 4.2$ & 0.84 & 0.79 \\
\hline RVD1 (mm) & $31.1 \pm 4.0$ & $32.1 \pm 4.4$ & $32.3 \pm 4.7^{\star}$ & $33.5 \pm 3.4^{*}$ & 0.75 & 0.39 \\
\hline RA volume (mL) & $40.3 \pm 16.7$ & $42.6 \pm 16.1$ & $45.3 \pm 20.8^{*}$ & $45.1 \pm 14.7^{*}$ & 0.16 & 0.40 \\
\hline TR vmax (m/s) & $2.7 \pm 0.4$ & $2.5 \pm 0.87$ & $1.9 \pm 1.1^{*}$ & $1.7 \pm 1.1$ & 0.54 & 0.67 \\
\hline SPAP $(m m ~ H g)$ & $37.5 \pm 9.1$ & $31.7 \pm 13.7$ & $27.9 \pm 6.7^{\star}$ & $22.8 \pm 13.6$ & 0.76 & 0.62 \\
\hline $\mathrm{HR}\left(\min ^{-1}\right)$ & $69.6 \pm 12.3$ & $63.4 \pm 9.1$ & $72.5 \pm 13.7^{*}$ & $71.2 \pm 9.2^{*}$ & 0.22 & 0.75 \\
\hline
\end{tabular}

Paired $t$ test versus baseline.

${ }^{*} \mathrm{P}<0.05 ;{ }^{* *} \mathrm{p}<0.01 ;{ }^{* *} \mathrm{p}<0.001$.

†Independent samples t-test to compare degree of changes seen following AVR with that seen following MIAVR.

$\mathrm{RV}$, right ventricular: $\mathrm{A}^{\prime}$, late diastolic myocardial velocity; AVR, aortic valve replacement surgery; $E^{\prime}$, early diastolic myocardial velocity; ET, ejection time; HR, heart rate; MIAVR, minimally invasive aortic valve replacement surgery; PEP, pre-ejection period; POP, post-ejection period; RA, right atrial; RIMP, right ventricular index of myocardial performance; RVD1, RV basal linear dimension; RVOT prox, proximal RV outflow diameter; S, peak systolic myocardial velocity; SPAP, systolic pulmonary artery pressure; TR, tricuspid regurgitation.

\section{RV size and function}

The end-diastolic RV transversal inflow diameter and proximal RV outflow diameter was similar in both groups before and after surgery (table 2 ).

Prior to surgery, there was no difference in RV-LS and RV-LSR between the patients undergoing AVR or MIAVR. Following surgery, RV-LS was deteriorated in both groups, although without any significant difference between them. RV-LSR was deteriorated in patients treated with AVR comparing values from baseline to values post-surgery $(-1.7 \pm 0.3$ vs $-1.4 \pm 0.31 / \mathrm{s}, \mathrm{p}<0.01)$. However, RV-LSR was preserved in patients treated with MIAVR when comparing preoperative values with postoperative values $(-1.5 \pm 0.5$ vs $-1.5 \pm 0.41 / \mathrm{s}, \mathrm{p}=0.84)$ (figure 2).

At baseline, there was no difference in RVS between the patients undergoing AVR and patients undergoing MIAVR. After surgery, RVS declined in both groups, however, to a much lesser extent in the MIAVR group compared with the AVR group $(18.8 \%$ vs $36.6 \%$, $\mathrm{p}<0.001)$.

There was no significant difference in RV performance as assessed by RIMP between patients undergoing
AVR and MIAVR at baseline. The RIMP was prolonged in both groups following surgery, although without any significant difference between the groups. The postoperative alterations in different phases of one cardiac cycle from baseline in one patient from each group is shown in figure 3 .

Sixteen patients $(84.2 \%)$ in the AVR group and 15 patients $(78.9 \%)$ in the MIAVR-group presented with a mild degree of tricuspid regurgitation before surgery. After surgery, 15 patients (78.9\%) in the AVR group and 15 patients $(78.9 \%)$ in the MIAVR group presented with mild degree of tricuspid regurgitation. No patients had moderate or severe tricuspid regurgitation in none of the groups neither before surgery nor after surgery. Prior to surgery, pulmonary artery systolic pressure (PASP) was mildly elevated in patients undergoing AVR $(37.5 \pm 9.1 \mathrm{~mm}$ $\mathrm{Hg})$. However, there was no statistically significant difference in PASP between the AVR group and MIAVR group at baseline. After surgery, PASP was reduced to normal levels in both AVR group and MIAVR group and without any significant difference between the groups $(27.9 \pm 6.7$ vs $22.8 \pm 13.6, \mathrm{p}=0.62$ ). 

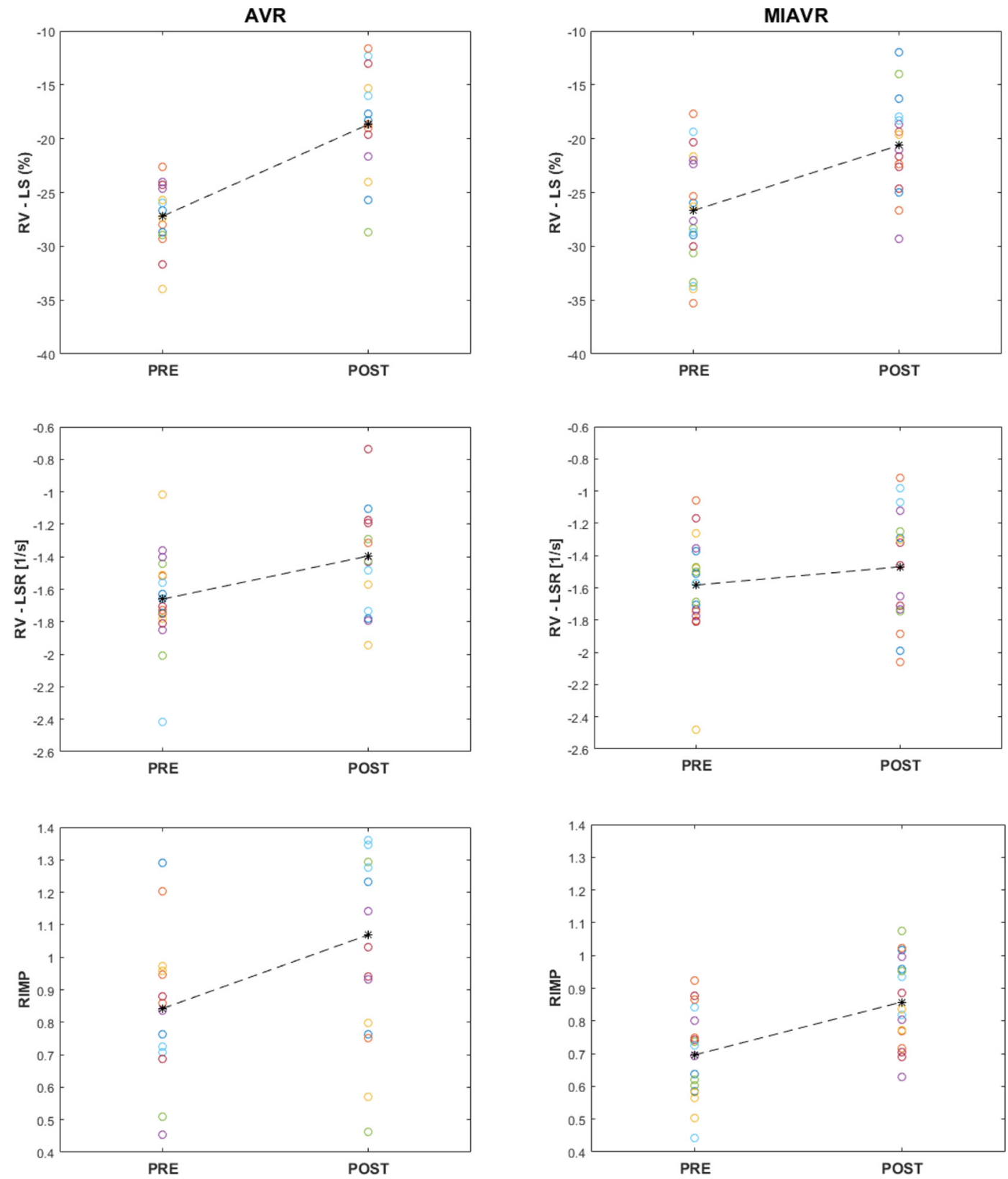

Figure 2 Changes in the parameters of right ventricular function in patients undergoing aortic valve replacement surgery (AVR) and minimally invasive aortic valve replacement surgery (MIAVR) comparing preoperative to postoperative values. RIMP, right ventricular index of myocardial performance; RV-FAC, right ventricular fractional area change; RV-LS, right ventricular longitudinal strain; RV-LSR, right ventricular longitudinal strain rate.

\section{LV size and function}

LV linear dimensions (LVEDD and LVESD) and LV volumes (LVEDV or LVESV) did not differ between the groups at baseline. LVEF was normal and without any difference when comparing patients undergoing AVR to patients undergoing MIAVR, both prior to surgery and postsurgery.

LV global longitudinal strain was within the normal range in both groups and remained within the normal range when comparing values and from baseline to post-surgery.

There was no difference in diastolic function between the AVR group and MIAVR group at baseline or after surgery (table 3 ).

\section{DISCUSSION}

The randomised, prospective CMILE study evaluated the impact of MIAVR and AVR on the regional and global RV function. In the present predefined post hoc analysis of the CMILE study, the principal findings were that MIAVR did not significantly affect RV intrinsic myocardial contractility assessed by RV-LSR, while both MIAVR and AVR induced decreased RV function when using methods that are more load dependent and dependent on extrinsic mechanical influence. RV contractile function is of significant clinical importance. It has been shown that RV contractility is one of the most important parameters for prediction of RV failure in patients treated with $\mathrm{LV}$ assist device and development of RV failure following 

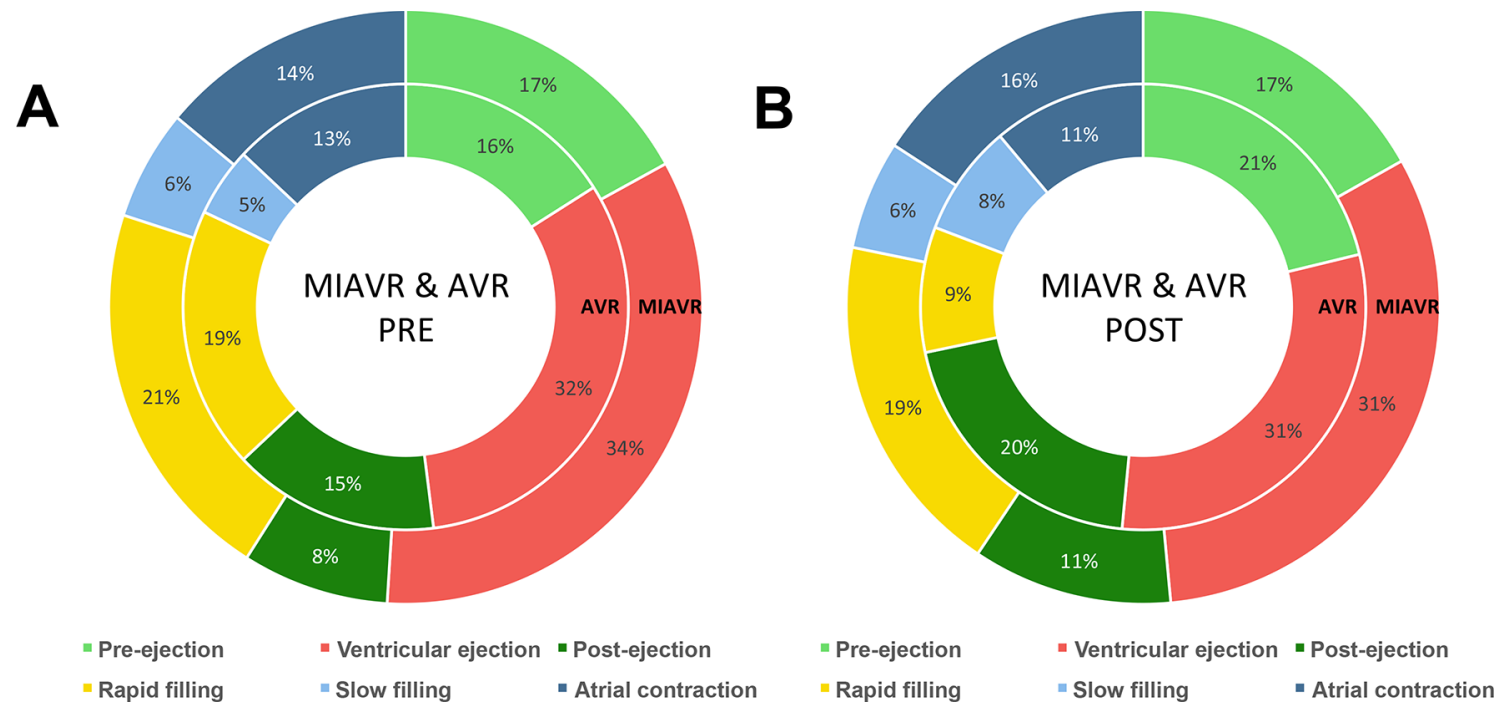

Figure 3 Example of right ventricular cardiac state diagram (CSD) displaying postoperative alterations in different phases of one cardiac cycle. AVR, aortic valve replacement surgery; MIAVR, minimally invasive aortic valve replacement surgery.

transplantation in patients with precapillary pulmonary hypertension. ${ }^{23}$ Furthermore, previous studies indicate that impaired RV contractile performance implicates poor outcomes in patients with valvular heart disease. ${ }^{24}$

Including more novel and conventional quantitative parameters of RV mechanics and contractility, we observed a significant reduction in regional and global RV function following AVR and MIAVR. Following surgery, RVS was reduced in both groups. However, the magnitude of reduction in RVS was significantly higher following AVR as compared with MIAVR, despite longer cardiopulmonary bypass time in the MIAVR group. Global RV function assessed by RV-LS and RIMP declined in both AVR and MIAVR without any significant difference between the groups. However, RV contractility assessed by RV-LSR was preserved following MIAVR while it was deteriorated following AVR. Previously, it has been acknowledged that load-dependent measures of RV function can change without changes in RV myocardial contractility. ${ }^{23}$

Table 3 Preoperative baseline and postoperative echocardiographic LV measurements in the two surgical groups

\begin{tabular}{|c|c|c|c|c|c|c|}
\hline \multirow[b]{2}{*}{ Variables } & \multicolumn{2}{|c|}{ Pre aortic valve surgery } & \multicolumn{2}{|c|}{ Post aortic valve surgery } & \multicolumn{2}{|l|}{ P values } \\
\hline & AVR & MIAVR & AVR & MIAVR & $\begin{array}{l}\text { Between } \\
\text { the groups } \\
\text { (preoperative) } \dagger\end{array}$ & $\begin{array}{l}\text { Between } \\
\text { the groups } \\
\text { (postoperative) } \dagger\end{array}$ \\
\hline LVEDD (mm) & $44.8 \pm 5.1$ & $46.3 \pm 5.3$ & $43.7 \pm 4.9^{*}$ & $47.1 \pm 5.6$ & 0.67 & 0.03 \\
\hline LVESD (mm) & $26.9 \pm 6.2$ & $28.7 \pm 6.9$ & $26.7 \pm 5.1$ & $30.6 \pm 6.9$ & 0.40 & 0.03 \\
\hline LVEDV (mL) & $85.6 \pm 19.9$ & $102 \pm 30$ & $82 \pm 17.2$ & $100.5 \pm 29.7$ & 0.14 & 0.03 \\
\hline LVEF (\%) & $60.2 \pm 5.7$ & $57.8 \pm 6.3$ & $56.5 \pm 5.4^{\star *}$ & $54.6 \pm 6.5$ & 0.33 & 0.37 \\
\hline LVGLS (\%) & $-16.5 \pm 8.1$ & $-17.4 \pm 2.1$ & $-15.7 \pm 2.7$ & $-16.9 \pm 2.1$ & 0.64 & 0.21 \\
\hline LVMPI & $0.81 \pm 0.3$ & $0.75 \pm 0.2$ & $0.91 \pm 0.2$ & $0.89 \pm 0.2^{\star *}$ & 0.37 & 0.63 \\
\hline Mitral E (m/s) & $0.81 \pm 0.3$ & $0.80 \pm 0.3$ & $0.84 \pm 0.2$ & $0.89 \pm 0.3$ & 0.72 & 0.71 \\
\hline LA volume (mL) & $83.2 \pm 23.3$ & $79.6 \pm 20.5$ & $75.2 \pm 22.1$ & $79.8 \pm 23.7$ & 0.53 & 0.58 \\
\hline $\mathrm{HR}\left(\min ^{-1}\right)$ & $69.6 \pm 12.0$ & $63.4 \pm 9.1$ & $72.5 \pm 13.7^{*}$ & $71.2 \pm 13.0^{*}$ & 0.22 & 0.75 \\
\hline
\end{tabular}

Paired $t$ test versus baseline.

${ }^{*} \mathrm{P}<0.05 ;{ }^{* *} \mathrm{P}<0.01 ;{ }^{* * *} \mathrm{P}<0.001$.

LV, left ventrucular: A, late diastolic flow velocity; AVR, conventional aortic valve replacement surgery; Dec T, declaration time; E, early diastolic flow velocity; EDD, end-diastolic diameter; EDV, end-diastolic volume; EF, ejection fraction; ESD, end-systolic diameter; ESV, endsystolic volume; GLS, global longitudinal strain; HR, heart rate; LA, left atrium; MIAVR, minimally invasive aortic valve replacement surgery; Mitral E/A, ratio between early diastolic flow velocity and late diastolic flow velocity: MPI, myocardial performance index. 
Our findings could provide an explanation for the discrepancies in earlier studies. The inconsistencies could thus be understood better from alterations in postoperative loading conditions and/or varying degrees of negative mechanical influence from the thoracotomy and pericardial manipulation. Incision of the pericardium due to altered pericardial constraint has been proposed to be the main factor responsible for deterioration of RV function. ${ }^{25}$ This hypothesis is further strengthened by our results revealing a postsurgical reduction in RVS and RV-LS following both AVR and MIAVR, despite the fact that during MIAVR only limited parts of the pericardium was incised, which was closed at the end of surgery. It can be speculated that RV is dependent on full pericardial support for maintaining its mechanical function, but this would not necessarily be due to the loss of myocardial contractility. Hence, opening even a small part of the pericardium may result in loss of that support. Furthermore, incision of the pericardium may result in geometrical changes of the $\mathrm{RV}$, which is not restored even after closing the pericardium. ${ }^{26}$ Although RV longitudinal function was more preserved in MIAVR as compared with AVR, no difference in global RV function was observed between the groups. Although RVS correlates well with global RV function in normal hearts, it does not correlate well with global RV function after open heart surgery. ${ }^{27}$ Increasing PASP has also been proposed to contribute to deterioration of RV function following AVR. In our study, we observed a reduction in PASP following aortic valve surgery regardless of surgical approach.

The effect of AVR on RV longitudinal function in our study is consistent with the results of previous studies. ${ }^{113}$ Currently, few data are available regarding the effect of MIAVR on RV function. In contrast to our study, Unsworth et $a l^{1}$ have reported preserved RV longitudinal function following MIAVR. This discrepancy could be explained by the fact that there were only eight patients included in that study. Furthermore, as the type of surgical approach for MIAVR has not been reported, it is difficult to compare the results of that study to ours, since the location and size of the pericardial incision has been suggested to affect the magnitude of post-surgical modifications in RV longitudinal contraction. ${ }^{28}$

To our knowledge, there are only a few studies available that used novel echocardiographic parameters comparing the impact of AVR and MIAVR on RV function in the context of RV mechanics and contractility. Although MIAVR was associated with a better preserved RVS, there was no significant difference between MIAVR and AVR in global RV function as assessed by RV-LS and RIMP. The state of postsurgical RV function can be translated into clinical outcomes. Dalen et al did not show any difference between AVR or MIAVR in 30-day mortality or 2-year survival. ${ }^{3}$

Our findings indicate a more complex picture, where myocardial contractility might be more preserved from MIAVR compared with AVR, but both MIAVR as well as AVR seem to negatively impact RV mechanical function despite preserved contractility in the MIAVR group. A composite of echo-derived parameters should be incorporated in assessment of RV function. Future studies are warranted for investigating long-term results of AVR and MIAVR on RV function and its clinical implications.

\section{Limitations}

The main limitation of this study is the relatively small number of patients included in the study. Also, the follow-up time of 40 days in our study was relatively short. Future studies with longer follow-up are required to assess long-term impact of MIAVR and AVR on RV function. Since our study is primarily an echocardiographic study, we would not be able to translate the echocardiographic differences between the groups into clinical outcomes, for example, exercise capacity.

\section{CONCLUSION}

Both MIAVR and AVR influence RV mechanical function, but MIAVR does not result in a decline in RV contractility. A composite of conventional and novel echocardiographic parameters should be incorporated in assessment for RV function since many estimates of RV function are load dependent.

\section{Author affiliations \\ ${ }^{1}$ Karolinska Institutet, Department of Clinical Sciences, Unit of Cardiology, Danderyd University Hospital, Stockholm, Sweden \\ ${ }^{2}$ Department of Clinical Physiology, Capio S:t Görans Hospital, Stockholm, Sweden ${ }^{3}$ Department of Medical Engineering, School of Technology and Health, KTH Royal Institute of Technology, Stockholm, Sweden \\ ${ }^{4}$ Heart and Vascular Theme, Karolinska University Hospital, Stockholm, Sweden \\ ${ }^{5}$ Karolinska Institutet, Department of Molecular Medicine and Surgery, Stockholm, Sweden \\ ${ }^{6}$ Department of Cardiology, Karolinska University Hospital, Stockholm, Sweden}

Contributors All authors have read and approved the manuscript. All authors have also sufficiently contributed to conception and design of the study, analysis and interpretation of data, drafting of the manuscript or revising it to justify authorship.

Funding NH received a grant from Capio Forskningsstiftelsen, grant number 20172995.

Competing interests None declared.

Patient consent Not required.

Ethics approval The study was approved by regional ethical committee in Stockholm, Sweden.

Provenance and peer review Not commissioned; externally peer reviewed.

Open access This is an open access article distributed in accordance with the Creative Commons Attribution Non Commercial (CC BY-NC 4.0) license, which permits others to distribute, remix, adapt, build upon this work non-commercially, and license their derivative works on different terms, provided the original work is properly cited and the use is non-commercial. See: http://creativecommons.org/ licenses/by-nc/4.0/

(c) Article author(s) (or their employer(s) unless otherwise stated in the text of the article) 2018. All rights reserved. No commercial use is permitted unless otherwise expressly granted.

\section{REFERENCES}

1. Unsworth B, Casula RP, Yadav H, et al. Contrasting effect of different cardiothoracic operations on echocardiographic right ventricular long axis velocities, and implications for interpretation of postoperative values. Int J Cardiol 2013;165:151-60. 
2. Gilmanov D, Solinas M, Farneti PA, et al. Minimally invasive aortic valve replacement: 12-year single center experience. Ann Cardiothorac Surg 2015;4:160-9.

3. Dalén M, Biancari F, Rubino AS, et al. Aortic valve replacement through full sternotomy with a stented bioprosthesis versus minimally invasive sternotomy with a sutureless bioprosthesis. Eur $J$ Cardiothorac Surg 2016;49:220-7.

4. Brown ML, McKellar SH, Sundt TM, et al. Ministernotomy versus conventional sternotomy for aortic valve replacement: a systematic review and meta-analysis. J Thorac Cardiovasc Surg 2009;137:670-9.

5. de Groote P, Millaire A, Foucher-Hossein C, et al. Right ventricular ejection fraction is an independent predictor of survival in patients with moderate heart failure. J Am Coll Cardiol 1998;32:948-54.

6. Di Salvo TG, Mathier M, Semigran MJ, et al. Preserved right ventricular ejection fraction predicts exercise capacity and survival in advanced heart failure. J Am Coll Cardiol 1995;25:1143-53.

7. Haddad F, Denault AY, Couture P, et al. Right ventricular myocardial performance index predicts perioperative mortality or circulatory failure in high-risk valvular surgery. J Am Soc Echocardiogr 2007;20:1065-72.

8. Kjaergaard J, Snyder EM, Hassager C, et al. Impact of preload and afterload on global and regional right ventricular function and pressure: a quantitative echocardiography study. J Am Soc Echocardiogr 2006;19:515-21.

9. Boldt J, Zickmann B, Ballesteros M, et al. Right ventricular function in patients with aortic stenosis undergoing aortic valve replacement. $J$ Cardiothorac Vasc Anesth 1992;6:287-91.

10. Tan TC, Flynn AW, Chen-Tournoux A, et al. Risk Prediction in Aortic Valve Replacement: Incremental Value of the Preoperative Echocardiogram. J Am Heart Assoc 2015;4:e002129.

11. Kempny A, Diller GP, Kaleschke G, et al. Impact of transcatheter aortic valve implantation or surgical aortic valve replacement on right ventricular function. Heart 2012;98:1299-304.

12. Duncan AE, Sarwar S, Kateby Kashy B, et al. Early Left and Right Ventricular Response to Aortic Valve Replacement. Anesth Analg 2017;124:406-18

13. Keyl C, Schneider J, Beyersdorf F, et al. Right ventricular function after aortic valve replacement: a pilot study comparing surgical and transcatheter procedures using 3D echocardiography. Eur $J$ Cardiothorac Surg 2016;49:966-71.

14. Missant C, Rex S, Claus $P$, et al. Load-sensitivity of regional tissue deformation in the right ventricle: isovolumic versus ejection-phase indices of contractility. Heart 2008;94:e15.

15. D'hooge J, Heimdal A, Jamal F, et al. Regional strain and strain rate measurements by cardiac ultrasound: principles, implementation and limitations. Eur J Echocardiogr 2000;1:154-70.
16. Dalén M, Oliveira Da Silva C, Sartipy U, et al. Comparison of right ventricular function after ministernotomy and full sternotomy aortic valve replacement: a randomized study. Interact Cardiovasc Thorac Surg 2018;26:790-7.

17. Nishimura RA, Otto CM, Bonow RO, et al. AHA/ACC guideline for the management of patients with valvular heart disease: a report of the American College of Cardiology/American Heart Association Task Force on Practice Guidelines. J Thorac Cardiovasc Surg 2014;2014:e1-e132.

18. Lang RM, Badano LP, Mor-Avi V, et al. Recommendations for cardiac chamber quantification by echocardiography in adults: an update from the American Society of Echocardiography and the European Association of Cardiovascular Imaging. Eur Heart J Cardiovasc Imaging 2015;16:233-71.

19. Quiñones MA, Otto CM, Stoddard M, et al. Recommendations for quantification of Doppler echocardiography: a report from the Doppler Quantification Task Force of the Nomenclature and Standards Committee of the American Society of Echocardiography. J Am Soc Echocardiogr 2002;15:167-84.

20. Larsson M, Bjällmark A, Johnson J, et al. State diagrams of the heart-a new approach to describing cardiac mechanics. Cardiovasc Ultrasound 2009;7:22.

21. Lundbäck S, Johnson J, Bergholm F, et al. Stockholm, SE), assignee. A cardiac state monitoring system. Sweden 2016.

22. Lundbäck S, iGHAS JJ. SE), assignee. State space model of a heart. United States 2012.

23. Dandel M, Hetzer R. Echocardiographic assessment of the right ventricle: Impact of the distinctly load dependency of its size, geometry and performance. Int J Cardiol 2016;221:1132-42.

24. Sade LE, Ozin B, Ulus T, et al. Right ventricular contractile reserve in mitral stenosis: implications on hemodynamic burden and clinical outcome. Int J Cardiol 2009;135:193-201.

25. Unsworth B, Casula RP, Kyriacou AA, et al. The right ventricular annular velocity reduction caused by coronary artery bypass graft surgery occurs at the moment of pericardial incision. Am Heart $J$ 2010;159:314-22.

26. Lindqvist P, Holmgren A, Zhao Y, et al. Effect of pericardial repair after aortic valve replacement on septal and right ventricular function. Int J Cardiol 2012;155:388-93.

27. Portnoy SG, Rudski LG. Echocardiographic evaluation of the right ventricle: a 2014 perspective. Curr Cardiol Rep 2015;17:21.

28. Okada DR, Rahmouni HW, Herrmann HC, et al. Assessment of right ventricular function by transthoracic echocardiography following aortic valve replacement. Echocardiography 2014;31:552-7. 